\title{
Meyer Neldel rule application to silicon supersaturated with transition metals
}

\author{
Eric García-Hemme ${ }^{1,2}$, Rodrigo García-Hernansanz $z^{1,2}$, Javier Olea ${ }^{2,3}$, David \\ Pastor, ${ }^{1,2,4}$, Alvaro del Prado ${ }^{1,2}$, Ignacio Mártil ${ }^{1,2}$ and Germán González-Díaz ${ }^{1,2}$ * \\ ${ }^{1}$ Dept. de Física Aplicada III (Electricidad y Electrónica), Univ. Complutense de Madrid, \\ 28040 Madrid, Spain \\ ${ }^{2}$ CEI Campus Moncloa, UCM-UPM, 28040 Madrid, Spain \\ ${ }^{3}$ Instituto de Energía Solar, E.T.S.I. de Telecomunicación, Univ. Politécnica de Madrid. \\ 28040 Madrid, Spain. \\ ${ }^{4}$ School of Engineering and Applied Sciences, Harvard University. Cambridge \\ Massachusetts 02138 USA. \\ * Author for correspondence germang@ucm.es
}

\begin{abstract}
This paper presents the results for the transverse conductance across a bilayer formed by supersaturating with diverse transition metals a thin layer of a silicon wafer. The layer is formed by ion implantation and annealed by pulsed laser melting. The transverse conductance is exponentially activated, obtaining values ranging from 0.018 to $0.7 \mathrm{eV}$ for the activation energy and pre-exponential factors of $10^{-}$ ${ }^{2}-10^{12} \mathrm{~S}$ depending on the annealing energy density. A semi-logarithmic plot of the pre-exponential factor versus activation energy shows an almost perfect linear behavior as stated by the Meyer Neldel rule. The Meyer Neldel energy obtained for implantation with different transition metals and also annealed in different conditions is $22 \mathrm{meV}$, which is within the range of silicon phonons, thus confirming the hypothesis of the Multi Excitation Entropy theory.
\end{abstract}

Keywords: Meyer-Neldel, supersaturated silicon, intermediate band, ion implantation

\section{I.- Introduction}

Silicon supersaturated with transition metals or chalcogens is becoming an important topic of study. In 1997, A. Luque and A. Martí [1] proposed the inclusion of deep levels in a semiconductor with enough density to surpass the Mott limit [2] to create a new band that was named intermediate band. Their proposal was intended for the improvement of solar cells. This improvement has not been proved yet, but the key operation principle has been demonstrated in intermediate band solar cells with quantum dots [3] and in highly mismatched alloys [4]. Also sub-bandgap external quantum efficiency has been reported in Ti supersaturated Si solar cells [5]. Otherwise supersaturation of $\mathrm{Si}$ with different elements like S [6], V[7] or $\mathrm{Au}$ [8] shows the capability to detect infrared radiation below the semiconductor gap energy, a fact that is undoubtedly related with the new levels introduced by the foreign elements.

The incorporation of supersaturating techniques to the very mature Si microelectronic technology will allow us to build a room temperature Si focal plane array infrared detector, a very promising device both in the civil and military fields.

Supersaturation, by definition, has to be obtained without the use of equilibrium techniques, being ion implantation (II) and pulsed laser melting (PLM) two of the out of equilibrium techniques most widely used. Nevertheless, the process of supersaturation is not yet well understood. In this respect the interface between the implanted layer and the substrate has to 
be studied more in depth. Implantation and PLM do not produce a perfectly abrupt impurity profile and besides crystallinity is not well defined in the border between the layer and the substrate. Both are points of concern.

The determination of the conduction mechanism between the supersaturated layer (implanted) and the silicon substrate is a key factor to understanding the transport properties of the bilayer and its possible uses. This conduction mechanism could be a current limitation due to a potential barrier. Most of the conduction processes in a semiconductor, and particularly at energy barriers, are exponentially dependent on temperature and consequently follow a typical Arrhenius equation as $G=G_{0} e^{-\frac{\Delta E}{k T}} \quad$ where $\mathrm{G}$ is, as an example, a conductance, $\Delta \mathrm{E}$ is the activation energy, $\mathrm{T}$ is the absolute temperature and $\mathrm{k}$ is the Boltzmann constant.

In the year 1937 Meyer and Neldel (MN) [9] found experimentally an exponential relation between $\mathrm{G}_{0}$ and $\Delta \mathrm{E}$, i.e. $G_{0}=G_{00} e^{b \Delta E}$ for a series of homogeneous samples with different activation energy $\Delta \mathrm{E}$, which, since then, is known as the MN rule (MNR). MNR is frequently written as $G_{0}=G_{00} e^{\frac{\Delta E}{k T_{m n}}}$ where $\mathrm{T}_{\mathrm{mn}}$ is called $\mathrm{MN}$ temperature and $\mathrm{kT}_{\mathrm{mn}}$ the $\mathrm{MN}$ energy. That rule, which appears in a wide variety of processes, is also known as the "compensation rule" in the chemistry field and predicts an isokinetic temperature for $\mathrm{T}=\mathrm{T}_{\mathrm{mn}}$ i.e. a temperature where the conductance for all the samples has the same value.

In the case of semiconductor barriers or junctions there are reports of MNR regarding the reverse characteristics of solar cells [10] or even regarding the forward characteristics of a silicon diode [11]. Some references try to explain this fact as due to a particular distribution of traps [12], but the universality of the process points toward a more fundamental explanation, as is the Multi Excitation Entropy (MEE) theory [13]. It seems that the MNR occurs only in disordered systems, when the accumulation or annihilation of elementary excitations is necessary to produce the event [14], in our case the promotion of a carrier over the energy barrier. In this sense, this carrier activation needs the help of many elementary excitations which are, in this case, phonons. In a recent paper [15] Yelon argues that despite the effect appears only in disordered systems, $T_{m n}$ could not be used as a measurement of this disorder, but is however related to this elementary excitation energy.

In this paper we will prove that the conduction through the boundary between the supersaturated Si layer and the substrate obeys the MNR and we will find the characteristic temperature. Also we will find that the $\mathrm{T}_{\mathrm{mn}}$ is not related with the implanted element, but is characteristic of the substrate itself.

\section{II.- Experimental}

Silicon substrates ( $\mathrm{N}$ type, $200 \Omega . \mathrm{cm}$ resistivity and $1450 \mathrm{~cm}^{2} / \mathrm{Vs}$ mobility) were implanted with $\mathrm{V}, \mathrm{Ti}, \mathrm{Zr}$ and $\mathrm{Cr}$ in doses of $10^{16} \mathrm{~cm}^{-2}$. The implantations were simulated with the Stopping and Range of Ions in Matter (SRIM) program, and the energies chosen to obtain the same depth profile for the different ions used ${ }^{48} \mathrm{Ti}$ at $32 \mathrm{KeV},{ }^{51} \mathrm{~V}$ and ${ }^{52} \mathrm{Cr}$ at $35 \mathrm{KeV}$ and ${ }^{90} \mathrm{Zr}$ at $49 \mathrm{KeV})$. Samples were implanted $7^{\circ}$ out of the normal incidence to reduce channeling effects. PLM processes were performed at I. P. G. Photonics (New Hampshire, USA). PLM was conducted in air, using a $\mathrm{KrF}$ excimer laser $(248 \mathrm{~nm})$, with a $20 \mathrm{~ns}$ single pulse and energy 
densities of $0.6,0.8,1$ and $1.2 \mathrm{~J} / \mathrm{cm}^{2}$. After PLM we subjected some Ti implanted samples to a Rapid Thermal Annealing (RTA) process in Ar atmosphere. We used a Heatpulse RTP-600 system (MPT Corporation Santa Clara, CA) and did 5 minutes isochronous consecutive annealings at 200,300 and $400^{\circ} \mathrm{C}$.

For the assessment of the implantation profile and in order to verify the PLM effect we performed Time of Flight Secondary Ion Mass Spectrometry (ToF-SIMS) measurements in a ToF-SIMS IV model manufactured by ION-TOF, with a $25 \mathrm{keV}$ pulsed $\mathrm{Bi}^{3+}$ beam at $45^{\circ}$ incidence. A $10 \mathrm{keV}$ voltage was used to extract the secondary ions generated and their time of flight from the sample to the detector was measured with a reflection mass spectrometer.

We measured the transport properties of the bilayer formed by the implanted layer and the substrate using the van der Paw configuration. Samples area is $1 \mathrm{~cm}^{2}$ and have electron beam evaporated triangular titanium $(100 \mathrm{~nm}) /$ aluminum $(200 \mathrm{~nm})$ contacts in the four corners. For sheet conductance measurements we introduce $1 \mathrm{~mA}$ at two contiguous contacts and we measure potentials at the 4 corners with a Keithley 4200 fitted with 4 source-measure units. Having the voltage at the four corners instead of just the difference between the two opposite corners allows us to detect contact problems [16]. Hall effect, using a 0.8T magnetic flux, was also measured with the same configuration and the same current but introducing it at opposite corners. Transport measurements were performed in the $10 \mathrm{~K}-300 \mathrm{~K}$ range in a Janis closed cycle cryostat with sample temperature control below $0.1 \mathrm{~K}$. We combined contacts, current and magnetic field in all possible ways in order to avoid errors due to spurious thermogalvanomagnetic effects. An unimplanted sample is also measured to obtain the precise data to characterize the substrate

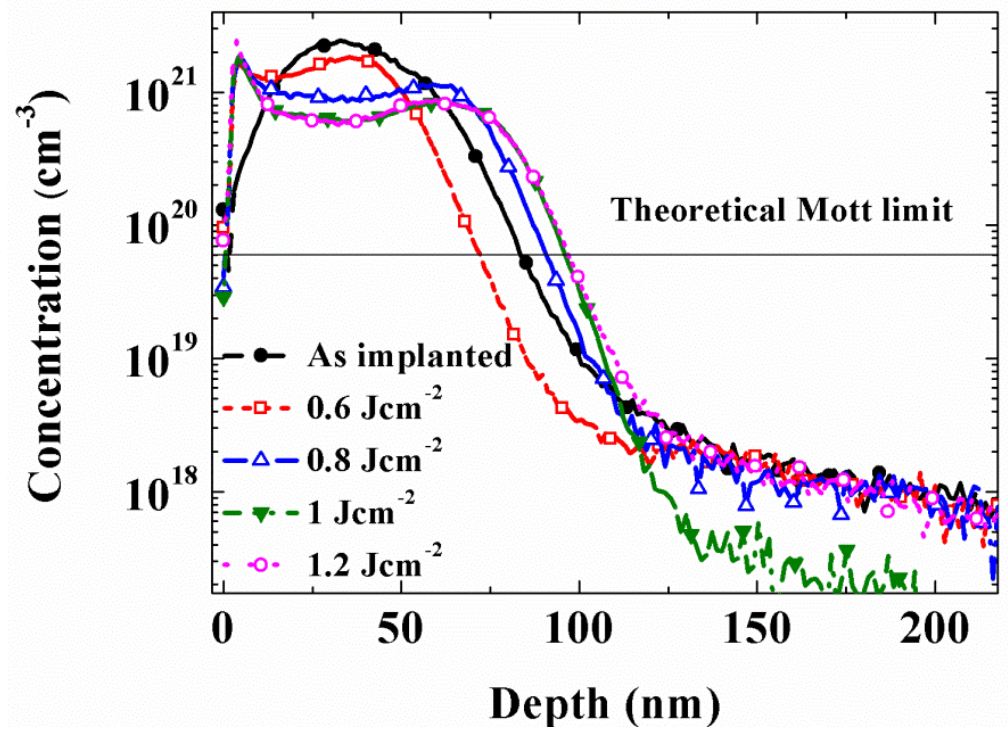

FIG.1. ToF-SIMS profiles of V-implanted samples with $10^{16} \mathrm{~cm}^{-2}$ dose after PLM annealing at $0.6,0.8,1$ and $1.2 \mathrm{~J} / \mathrm{cm} 2$. The implanted but non-annealed sample is also shown. Lines represent data while the symbols are to guide the eye 


\section{III.- Results}

The data that we will present for electrical transport properties and for SIMS profiles in the next paragraphs are those for V implanted samples. Samples implanted with other ions ( $\mathrm{Zr}$, $\mathrm{Ti}$ and $\mathrm{Cr}$ ) show very similar behaviors to these and are not included in the graphs for the sake of clarity.

Fig. 1 presents the $\mathrm{V}$ profile before and after PLM processes at the 4 energy densities used. The horizontal line is the Mott limit, which was studied from a theoretical point of view in reference [17] and experimentally in reference [18]. While this limit could not be calculated or measured precisely, our experimental data confirm that it value is lower but closer to $10^{20} \mathrm{~cm}^{-3}$ for our particular case study. The PLM produces a clear V redistribution, with layers from 75 to $100 \mathrm{~nm}$ with V concentration over the Mott limit. Below this thickness, V concentration ranges from $6 \times 10^{20} \mathrm{~cm}^{-3}-2 \times 10^{21} \mathrm{~cm}^{-3}$ depending on the sample. The peak close to the surface is believed to be a SIMS artifact.

Sheet conductance is presented on Fig. 2(a) for the 4 different annealing processes and also for the unimplanted substrate. The Hall mobility for the same samples is presented in Fig. 2(b). In the following, and for clarity, electron mobility is assumed to be positive while hole mobility is consequently assumed to be negative. In both cases symbols stand for the experimental data, and the continuous lines for the simulation, as explained below. From Fig. 2(a) it is clear that at high temperature the bilayer conductance is higher than the substrate conductance, as it corresponds to the parallel behavior of two conductive layers. As the temperature decreases, and depending on the PLM energy density, the bilayer conductance crosses the substrate conductance and remains below it. This is an odd behavior for a parallel set of conductive layers and implies a decoupling between the upper layer and the substrate. Below a PLM dependent critical temperature, the implanted layer starts the process of isolation and becomes totally isolated when the conductance is almost temperature independent. This fact allows us to determine the electrical parameters of this layer, i.e. the carrier concentration and its mobility. For very low temperatures the substrate carriers freeze out and the substrate conductance drops to zero, but this does not affect the upper layer conductance, already fully decoupled.

We fit the data to a model of bilayer transport behavior previously developed [19, 20] that takes into account the transport parameters of the implanted layer, the substrate parameters and an energy barrier that limits the current between the upper (implanted) layer and the substrate. The data in Fig. 2(a) are fitted to the following expression for the sheet conductance:

$$
G_{\text {sheet }}=\frac{\left(G_{i m}+G_{s u b} F\right)^{2}}{G_{i m}+G_{s u b} F^{2}}
$$

Where $\mathrm{G}_{\mathrm{im}}$ is the sheet conductance of the implanted layer, $\mathrm{G}_{\text {sub }}$ is the sheet conductance of the substrate and $\mathrm{F}$ is the decoupling factor that takes into account the current limitation between the upper layer and substrate. The $\mathrm{F}$ factor is formulated as:

$$
F=\frac{G_{t}}{G_{t}+G_{s u b} / \alpha}
$$



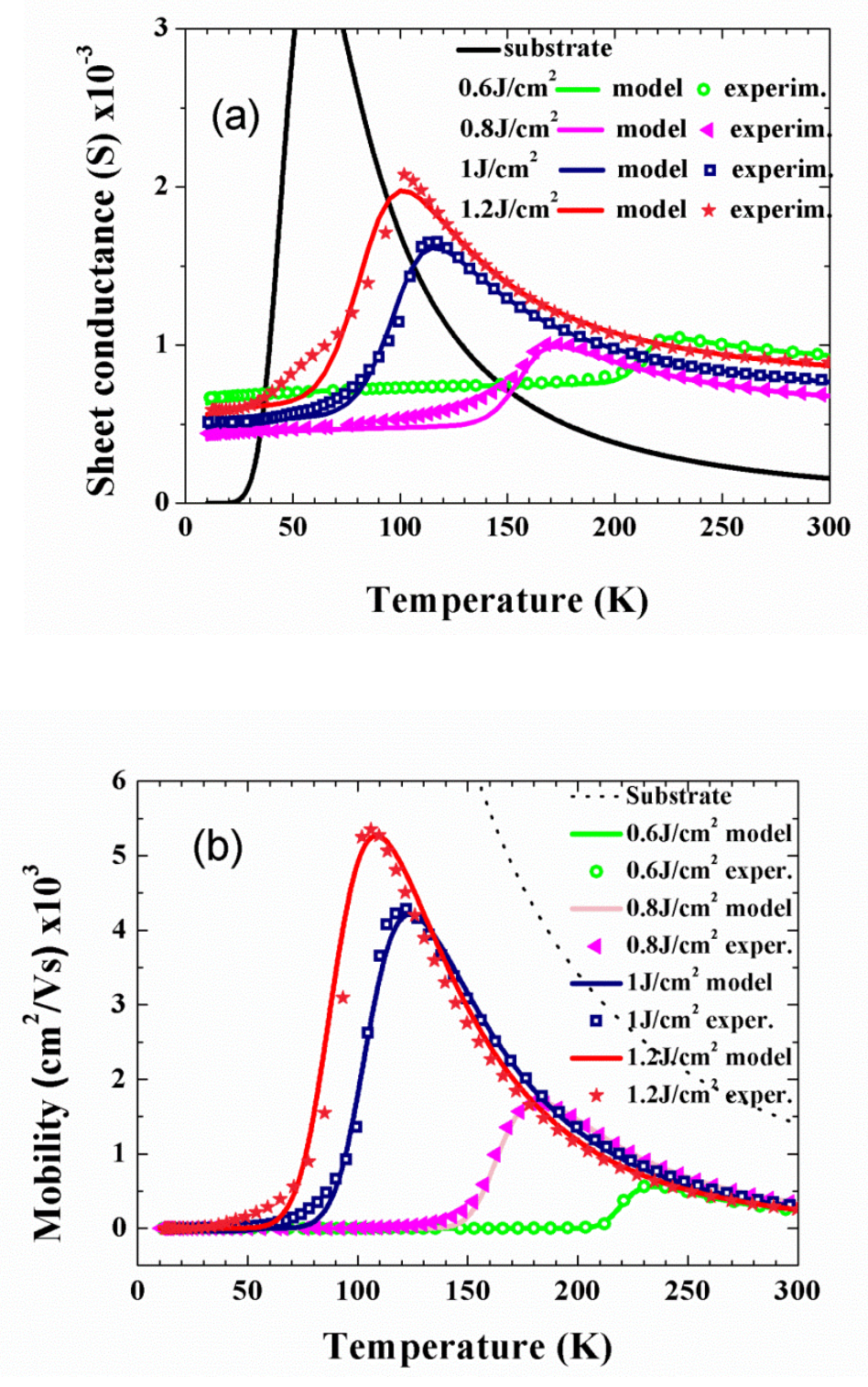

FIG. 2.(a) Measured sheet conductance and sample modelling (Gsheet) as a function of measurement temperature. (b) Measured and modelled ( $\mu$ eff ) Hall mobility.

Where $\alpha$ is a factor that takes into account the ratio between contact size and sample size and $G_{t}$ is the transverse conductance associated with the limiting barrier which is modelled as exponentially dependent on temperature as:

$$
G_{t}=G_{0}^{-\Delta E / k T}
$$

F function ranges from 0 at low temperature when $\mathrm{G}_{\mathrm{t}}$ is very low to 1 at high temperature when the transverse conductance is higher than the substrate conductance. In the first case, very few carriers can surpass the $\Delta \mathrm{E}$ barrier and, as a consequence, the implanted layer is electrically decoupled from the substrate. In the second case, both layers are in parallel, and there is no limitation in the carrier flow between the implanted layer and the substrate. 
Bilayer mobility could also be found as a function of the mobilities and conductances of both layers and the final expression is

$$
\mu_{e f f}=\frac{\mu_{i m} G_{i m}+\mu_{s u b} G_{s u b} F^{2}}{G_{i m}+G_{s u b} F^{2}}
$$

where $\mu_{\mathrm{im}}$ and $\mu_{\text {sub }}$ are respectively the carrier dominant mobilities of the implanted layer and of the substrate. F and the conductances have the same meaning as in Eq (1). $\mu_{\text {im }}$ gives us an insight into the characteristics of the implanted layer. For all the V samples, irrespective of the annealing energy density, its value and temperature dependence can be modelled as $\mu_{\mathrm{im}}=$ $0.1(\mathrm{~T} / 300)^{0.07}$ which is consistent with a semi-metalic behavior. For other elements there are small variations in the room temperature mobility.

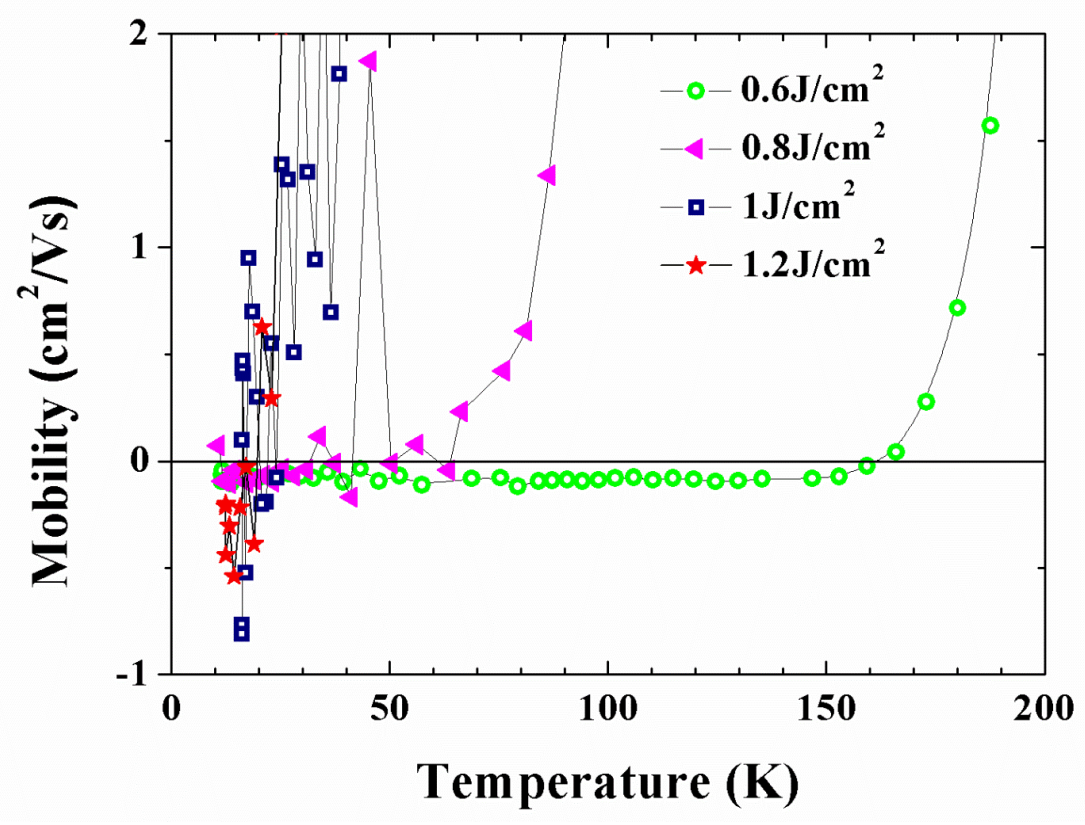

FIG.3. Mobility data of samples of Fig. 2 (b) in the low temperature zone

As it can be seen in Fig. 2(a) and 2(b) the fittings are very precise, mainly in the mobility plot. Going from the coupled state to the decoupled one (from high temperature to low temperature) we can find a maximum both in the conductance and in the mobility that depends on the annealing energy density. Well below this critical temperature $T_{c}$ the model predicts that the transport parameters have to be entirely dependent on the implanted layer. The mobility in Fig. 2(b) determines that the electrons are the dominant carriers in the coupled zone. In Fig. 3 we represent, with the same symbols used in Fig. 2(b), a zoom of the mobility plot to show that the mobility has changed its sign and now, at the implanted layer, the dominant carriers are holes with very low mobility, in the order of $0.1 \mathrm{~cm}^{2} / \mathrm{Vs}$. This effect is more evident for samples annealed at 0.6 and $0.8 \mathrm{~J} / \mathrm{cm}^{2}$ which become decoupled during a wider temperature interval, while 
samples annealed at higher energies are noisy. The same change of sign takes place when we implant other ions

The good fitting of the experimental sheet conductance and Hall mobility to the model (Fig. 2(a) and 2(b)) allows us to deduce the carrier density (holes) at the implanted layer. Carrier concentration and sheet conductance for this layer are presented in Table I for the four PLM energies used. The fact that the carriers for low temperatures are holes does not imply that the current limitation between the implanted layer and the substrate could be assimilated to a $p-n$ junction. This is because the $p$ type carriers at the IB can't cross to the substrate because there is not continuity between this band and any other in the substrate. The idea of the IB isolation is essential for solar cell devices. Our only assumption in relation to the transverse conduction is that it is limited in an exponential way. Due to the implantation tails, the physics under this limitation seems to be more related to a large density of states in the border between the implanted layer and the substrate than to a real p-n junction understood as two semiconductors with different Fermi levels that should be equalized.

Fig. 2(b) shows that the bilayer mobility in the coupled zone is a fraction of the substrate mobility, and almost independent of the PLM energy density. This result is easily understood if we realize that for this zone the conductance $G_{t}$ has to be very high, the $F$ factor is almost 1 , and the effective mobility could be written as:

$$
\mu_{e f f} \approx \frac{\mu_{i m} G_{i m}+\mu_{s u b} G_{s u b}}{G_{i m}+G_{s u b}} \approx \frac{\mu_{s u b}}{1+G_{i m} / G_{s u b}}
$$

because $\mathrm{G}_{\mathrm{im}}$ and $\mathrm{G}_{\text {sub }}$ are of the same order and $\mu_{\mathrm{im}}<<\mu_{\text {sub. }}$. As the conductance of the implanted layer is almost independent of the PLM process, all the mobility curves also have to be almost coincident as long as the layers remain coupled.

The fact that the conductances of the implanted layers (below the critical temperature) do not present the freeze-out effect, unlike the substrate, implies a semimetalic behavior of this layer which in turn implies a pinned Fermi level and a finite conductance at very low temperatures. The absence of the freeze out effect for these samples has been studied in detail in Ref. [21].

The best fitting values for the pre-exponential factor of $\mathrm{G}_{t}$ i.e. $\mathrm{G}_{0}$ and the blocking energy $\Delta \mathrm{E}$ are strongly dependent on the annealing process. For the $\mathrm{V}$-implanted samples of Fig. 2(a) and (b), we obtain the results presented on Table I. The temperatures for maximum mobility are also listed. A striking point is the hole concentration which is, higher than the vanadium concentration as it can be seen if we compare the data in table 1 with figure 1 . Two facts can be pointed out as an explanation;

- the Hall factor " $r$ " was assumed to be 1, but to calculate the exact carrier concentration we should have known the exact value. This factor is very dependent on the E-k band shape which is unknown in our case.

- as we are dealing with a new extra band and not with the case of simple donors or acceptors we have to admit the possibility of having filled as well as empty states that behaves as either electrons or holes depending on the curvature of the E-k relationship 


\begin{tabular}{cccccc}
\hline \hline $\begin{array}{c}\text { PLM Energy density } \\
\left(\mathrm{J} / \mathrm{cm}^{2}\right)\end{array}$ & $\mathrm{p}\left(\mathrm{cm}^{-3}\right)$ & $\mathrm{G}_{\mathrm{im}}(\mathrm{S} / \square)$ & $\mathrm{G}_{0}(\mathrm{~S})$ & $\Delta \mathrm{E}(\mathrm{eV})$ & $\mathrm{T}_{\mathrm{c}}(\mathrm{K})$ \\
\hline 0.6 & $6.75 \times 10^{21}$ & $7.7 \times 10^{-4}$ & $2.50 \times 10^{12}$ & 0.7 & 235 \\
0.8 & $3.55 \times 10^{21}$ & $5.17 \times 10^{-4}$ & $2.94 \times 10^{3}$ & 0.263 & 180 \\
1 & $4.10 \times 10^{21}$ & $6.1 \times 10^{-4}$ & $4.44 \times 10^{1}$ & 0.065 & 126 \\
1.2 & $4.80 \times 10^{21}$ & $7.14 \times 10^{-4}$ & $8,70 \times 10^{-2}$ & 0.042 & 105 \\
\hline \hline
\end{tabular}

TABLE I: PLM energy density, implanted layer carrier concentration (holes), implanted layer sheet conductance, blocking conductance pre-exponential factor, Arrhenius energy and decoupling temperature for V implanted samples

To check the influence of the implanted metal on the transverse blocking conductance we measured similar bilayers built in the same silicon substrate, but implanted with $\mathrm{Zr}, \mathrm{Cr}$ and $\mathrm{Ti}$ at $10^{16} \mathrm{~cm}^{-2}$ dose and PLM annealed at the same energy densities than the V implanted ones: 0.6 , $0.8,1$ and $1.2 \mathrm{~J} / \mathrm{cm}^{2}$. The conductance and mobility curves have the same shape as the ones for $\mathrm{V}$ and the fitting procedure is as quoted above. In Fig. 4 we represent the MN plot, i.e a semilogarithmic plot of $\mathrm{G}_{0}$ as function of $\Delta \mathrm{E}$, for all the samples. As it can be seen, we have obtained an almost perfect linear fit. This linear dependence extends over 14 decades in the conductance axis. Some samples with other doses $\left(10^{15}\right.$ and $\left.5 \times 10^{15} \mathrm{~cm}^{-2}\right)$, over the Mott limit, have been measured giving the activation energy and the conductance pre-exponential factor that fit perfectly the data presented in fig 4 . We have chosen the set of samples with $10^{16} \mathrm{~cm}^{-2}$ dose just for completeness.

If we use the most common expression for the Meyer Neldel rule, i.e. a constant preexponential factor and a $\mathrm{MN}$ temperature $\left(\mathrm{T}_{\mathrm{MN}}\right)$ :

$$
G_{t}(T)=G_{00} e^{-\frac{\Delta E}{k}\left(\frac{1}{T}-\frac{1}{T_{M N}}\right)}
$$

we obtain as the best fit a value of $\mathrm{G}_{00}=2.11 \times 10^{-2} \mathrm{~S}$ and a Meyer Neldel temperature $\mathrm{T}_{\mathrm{MN}}=$ $256 \mathrm{~K}$. The energy corresponding to this temperature is $\Delta \mathrm{E}_{\mathrm{MN}}=\mathrm{k} \cdot \mathrm{T}_{\mathrm{MN}}=22 \mathrm{meV}$.

The influence of the PLM energy density is very clearly observed in Fig. 4 where four groups of data can be differentiated. For $0.6 \mathrm{~J} / \mathrm{cm}^{2}$ annealing energy density we obtain blocking energies ranging from $0.53 \mathrm{eV}-0.7 \mathrm{eV}$, for $0.8 \mathrm{~J} / \mathrm{cm}^{2}$ blocking energies from $0.25 \mathrm{eV}-0.253 \mathrm{eV}$, for $1 \mathrm{~J} / \mathrm{cm}^{2}$ from $0.065 \mathrm{eV}-0.09 \mathrm{eV}$, and finally for $1.2 \mathrm{~J} / \mathrm{cm}^{2}$ blocking energies ranged from $0.03 \mathrm{eV}$ $0.042 \mathrm{eV}$. We could not find any correlation between these energies and the implanted element.

A sample previously implanted with Ti at a dose of $10^{16} \mathrm{~cm}^{-2}$ at $20 \mathrm{keV}$ and PLM annealed at an energy density of $0.8 \mathrm{~J} / \mathrm{cm}^{2}$ was subjected to subsequent RTA processes of 5 minutes at 200 , 300 and $400^{\circ} \mathrm{C}$. Transverse conductances obtained from the fitting of measurements showed a consistent decrease of the blocking energy from $0.17 \mathrm{eV}$ for the PLM annealed to $0.039 \mathrm{eV}$ when the sample was also RTA annealed at $400^{\circ} \mathrm{C}$ and a decrease in the pre-exponential values. Those data are also presented in Fig. 4. The dashed line in this fig. represents the best fitting. 


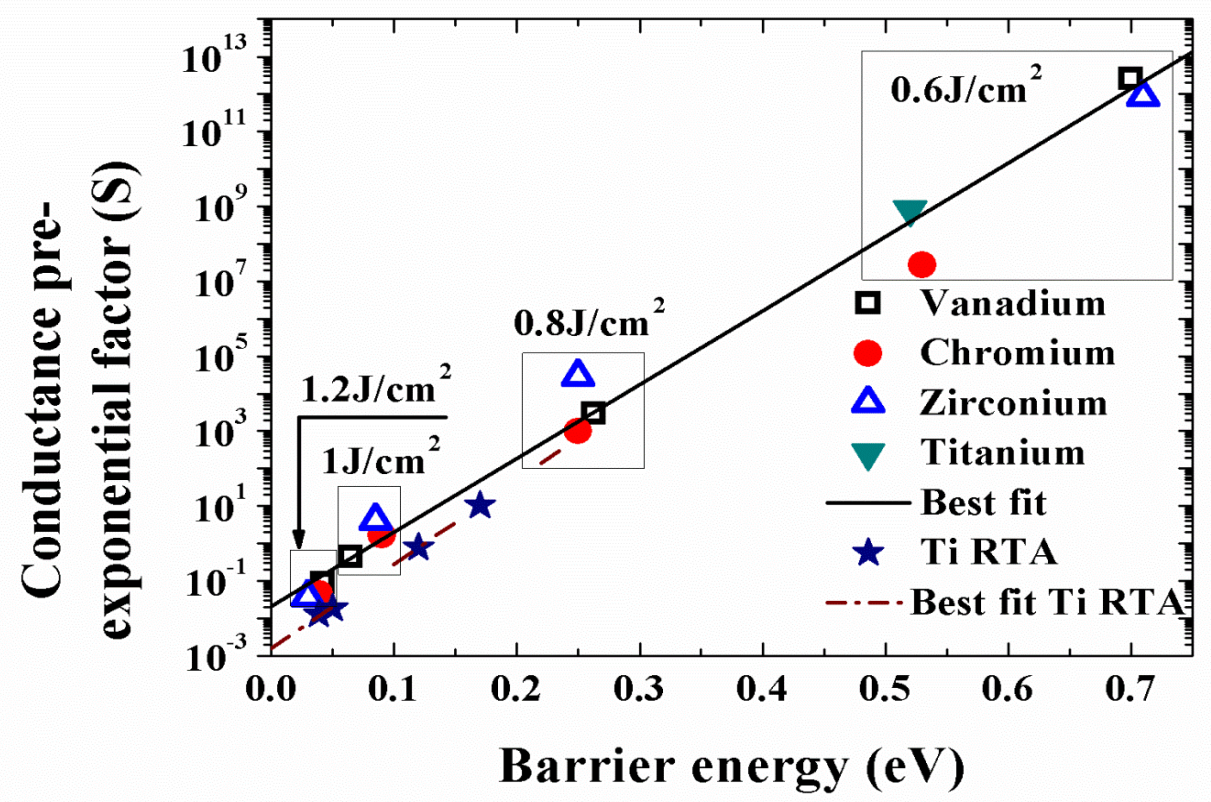

FIG. 4. Meyer Neldel plot for the samples implanted with all the transition metals analyzed: ( $\boldsymbol{\nabla}) \mathrm{Ti}$,

$\mathrm{Cr}$ and ( $\mathbf{\Delta}$ ) Zr. Continuous line is the best fitting for all the PLM samples. Meyer Neldel plot of a sample implanted with Ti and PLM annealed at $0.8 \mathrm{~J} / \mathrm{cm} 2$ and further heated in the RTA system at 200,300 and $400^{\circ} \mathrm{C}$ during $5 \mathrm{~min}(\star)$. Slotted line is the best fitting for PLM and RTA samples.

Now, $\mathrm{T}_{\mathrm{MN}}=224 \mathrm{~K}, \Delta \mathrm{E}_{\mathrm{MN}}=19 \mathrm{meV}$ and the pre-exponential factor $\mathrm{G}_{00}=1.6 \times 10^{-3} \mathrm{~S}$, values that are consistent with the data presented for the complete set of measurements. Consequently, the rapid thermal processing decreases the effect of the blocking transverse conductance but remains within the same $\mathrm{MN}$ plot. We think that the difference between the present $19 \mathrm{meV}$ and $22 \mathrm{meV}$ obtained before is due to inaccuracies in the measurements.

\section{IV.- Discussion}

After PLM, the implantation of doses of $10^{16} \mathrm{~cm}^{-2}$ of transition metals in silicon produces a layer of typical thickness of about $100 \mathrm{~nm}$ of the element implanted, with a concentration over the Mott limit. This layer is very conductive, with sheet conductance of about $6 \times 10^{-4} \mathrm{~S}$ and a $\mathrm{p}$ type mobility of $0.1 \mathrm{~cm}^{2} / \mathrm{Vs}$. Moreover this conductance does not present the freeze out effect unlike the silicon substrate, i.e. the implanted layer behaves as a semimetal having a pinned Fermi level. Those data come from fitting the model, but could also be obtained directly from measurements at low temperatures.

Considering the sheet conductance and the implanted thickness that have impurities over the Mott limit, we can obtain the implanted layer conductivity. Assuming an equal mobility of $0.1 \mathrm{~cm}^{2} /$ Vs for all the samples we can obtain the average carrier density for the different PLM energies, as reported in Table I. The differences in these values are within the expected uncertainty.

At doses of $10^{16} \mathrm{~cm}^{-2}$, the PLM process is not capable of producing a perfect recrystallization, and implanted layers are defective [22]. The degree of crystallization depends on the energy used during the PLM process, and the implanted layers are clearly better ordered 
as the energy grows. It has been argued [23] that the implanted element could remain trapped in defects or be accumulated unevenly due to the process known as cellular breakdown. As the carrier concentration remains almost constant (Table I), this effect in not determining, at least for the conductive properties of the films. Consequently the carrier concentration remains also constant regarding cristallinity. In Ref [19] and [20] we compare the carrier density for samples at Ti doses, of $10^{15}, 5 \times 10^{15}$ and $10^{16} \mathrm{~cm}^{-2}$, and this showed a perfect correlation between the doses and carrier concentration. This was so in spite of the very different crystallinity of the samples, which goes from almost perfect crystal (with scarce defects) at $10^{15} \mathrm{~cm}^{-2}$ to a defective film at $10^{16} \mathrm{~cm}^{-2}$ dose. The hypothesis of metal evenly distributed was confirmed by Raman measurement in a Ti implanted sample which does not show any vibration peak related with Ti-Ti bonds [24]. At least in the case of Ti, RBS analysis showed that the metal is incorporated as interstitial in the Si network [25].

It is a key question that this conductive layer appears only when the implanted concentration is over the Mott limit as it was proved in Ref. [18]. When the concentration is below (doses under $10^{14} \mathrm{~cm}^{-2}$ ), there is not any conductance in the implanted layer and the conductance and mobility measurements are almost indistinguishable from those of the substrate.

Unfortunately it is impossible to obtain an infinitely abrupt metal profile by implantation because tails of the implanted element with concentration below the Mott limit will remain at the border between the conductive layer and the substrate as shown in Fig. 1. As it is well known, transition metals in Si are very active recombination centers and this could be a possible origin of the transverse conductance limitation. We must bear in mind that in spite of having a concentration below the Mott limit, the transition metal concentration at this tail region, is high enough to pin the Fermi level to an unknown value.

In Ref [19] and [20], we attribute the decoupling effect to a difference between the Fermi level and the conduction band both in the implanted layer and in the substrate. The big variations in $\Delta \mathrm{E}$ and in $\mathrm{G}_{0}$ as the annealing energy density changes can't be associated to Fermi level displacements, and are probably more related to a limitation produced by a loss of crystallinity or to a high metal density (but lower than Mott limit) in the boundary between the implanted layer and the substrate. This limitation in the transversal conductivity could be more important than the difference between Fermi levels, and will conceal the effect of this difference. It is important to note that a hypothetical carrier going from an electrode to the other through the substrate has to cross the barrier twice, one from the implanted layer to the substrate and the other in a reverse sense.

In conclusion, we have a conductance limitation that we associate with some kind of disorder at the border between a supersaturated silicon layer, which behaves as a semimetal and a lightly doped n-type silicon wafer. Due to measuring configuration, we can't know if the conduction limitation is higher in the direction from the implanted layer to the substrate or vice versa.

It is known that for semiconductors the Meyer Neldel energy is related with the semiconductor band gap value, becoming higher as the forbidden gap widens [13]. For amorphous silicon this energy is around 50meV [26] while Coutts and Pearsall [10] found an energy of $31 \mathrm{meV}$ for the reverse current of solar cells made with semiconductors with gap comprised between 1 and $2 \mathrm{eV}$. Our energy is $22 \mathrm{meV}$, which is low, but in the order of the ones cited above. 
Pichon [27] gives MN energy values both for amorphous and polycrystalline silicon. In the first case the energy is $51 \mathrm{meV}$, while in the second case he found differences related with the deposition parameters, with the MN energy ranging from $41 \mathrm{meV}-63 \mathrm{meV}$.

Witanachchi et al [28] developed a very similar model to ours to account for the four points resistivity measurements of a bilayer of $\mathrm{FeSi}$ deposited over $\mathrm{Si}$, without avoiding the silicon native oxide. Through his model he obtained the thermal dependence of the resistance that limits the conduction between the FeSi conductive layer and the substrate. The pre-exponential factor obtained for the conductance was $8 \times 10^{8} \mathrm{~S}$ and the energy barrier $0.54 \mathrm{eV}$. According to our data, the pre-exponential factor associated to a value of the barrier of $0.54 \mathrm{eV}$ is $9.6 \times 10^{8} \mathrm{~S}$, in good agreement with Witanachchi value. Unfortunately the paper does not present data for other barrier energies, so it is not possible to find the $\mathrm{T}_{\mathrm{mn}}$ associated with the transversal conduction he obtained. Widenhorn et al [29] calculated the MNR for the dark current in a silicon CCD and found a MN energy of $25 \mathrm{meV}$, which is very close to our result.

The similarity between the Witanachchi conductance and the Widenhorn energy with our results points towards the fact that the $\mathrm{MN}$ rule is related not to a specific conduction problem, but to a more fundamental effect, as explained in the MEE theory. In all solid state conduction phenomena, according to this theory the $\mathrm{MN}$ energy has to be related to the phonon energies.(Ref [15] and [30])

Yelon, Movaghar and Branz [14] suggested that the MN rule breaks for energies below the MN energy, and would give lower values than expected. In our case it is difficult to observe this effect due to the low value of the MN energy but in Fig. 4 the three lower values of the energy seems to slightly deviate below the regression line.

\section{V.- Conclusions}

Using a previously developed model for the electrical conduction of a bilayer we have obtained the transport parameters associated with both layers and also with the conduction between the implanted and annealed upper layer and the substrate. We did that process for different energy density annealings, and also for different implanted ions, all of them transition metals. The model assumes that the limitation is due to a barrier that has to be surpassed and consequently is modelled as an Arrhenius conductance.

Plotting the pre-exponential factor versus the barrier energy we obtained a linear regression for a wide range of barrier energies. This confirms the $\mathrm{MN}$ rule. We could not find in the literature values for the MN energy for crystalline silicon, but the obtained value of $22 \mathrm{meV}$ is smaller though close to the one obtained for amorphous silicon. That similitude is in accordance with the MEE theory which establishes that the transitions involved in an MN event are directly related with the semiconductor energy gap.

Besides proving the validity of the MN rule in our bilayer, the good regression of the MN plot to a line validates the hypothesis related with the limitation of the conductivity in the bilayer boundary presented in the model. It is important to bear in mind the wide variety of conditions i.e. different annealing energy densities, different implanted elements (but all of them transition elements) and finally the post RTA annealing. Also, the very wide range for the conductance pre- 
exponential factor that runs over 14 orders of magnitude guarantees the validity of the assumptions made in the model.

\section{Acknowledgements}

Authors would like to acknowledge the CAI de Técnicas Físicas of the Universidad Complutense de Madrid for the ion implantations and metallic evaporations and to the Nanotechnology and Surface Analysis Services of the Universidad de Vigo C.A.C.T.I. for ToFSIMS measurements. This work was partially supported by the Project MADRID-PV (Grant No. P2013/MAE-2780) funded by the Comunidad de Madrid and by the Spanish MINECO (Economic and Competitiviness Ministery) under grant TEC 2013-41730-R

Research by E. García-Hemme was also supported by a PICATA predoctoral fellowship of the Moncloa Campus of International Excellence (UCM-UPM). J. Olea thank Professor A. Martí and Professor A. Luque for useful discussions and guidance and acknowledge financial support from the MICINN within the program Juan de la Cierva (JCI- 2011-10402 and JCI-201111471), under which this research was undertaken. D. Pastor acknowledges the financial support to the grant EX-2010-0662 from the Spanish Science Ministry 


\section{References}

[1] A. Luque and A. Marti. Phys. Rev. Lett. 78, 5014 (1997)

[2] N. F. Mott, Rev. Mod. Phys. 40, 677 (1968)

[3] A. Martí, E. Antolín, E. Cánovas, N. López, P.G. Linaes, A. Luque, C. Stanley and C. Farmer. Thin Solid Films 516, 6716 (2008)

[4] N. López, L. A. Reichertz, K. M. Yu, K. Campman, and W. Walukiewicz. Phys. Rev. Lett. 106, 028701 (2011)

[5] S. Silvestre, A. Boronat, M. Colina, L. Castañer, J. Olea, D. Pastor, A. del Prado, I. Mártil, G. GonzálezDíaz, A. Luque, E. Antolín, E. Hernández, I. Ramiro, I. Artacho, E. López and A. Martí. Jap. J. Appl. Phys. 52, $122302(2013)$

[6] J. T. Sullivan, C. B. Simmons, J. J. Krich, A. J. Akey, D. Recht, M. J. Aziz, and T. Buonassisi, , J. Appl. Phys. 114, 103701 (2013)

[7] E. García-Hemme, R. García-Hernansanz, J. Olea, D. Pastor, A. del Prado, I. Mártil and G. GónzalezDíaz Appl. Phys. Lett. 103, 032101 (2013)

[8] J. P. Mailoa, A. J. Akey, C. B. Simmons, D. Hutchinson, J. Mathews, J. T. Sullivan, D. Recht, M. T. Winkler, J. S. Williams, J. M. Warrender, P. D. Persans, M. J. Aziz and T. Buonassisi, Nature Comm. 5, 3011 (2014)

[9] W. Meyer and H. Neldel, Phys. Zeitsch. 38, 1014 (1937)

[10] T. J. Coutts and N. M. Pearsall. Appl. Phys. Lett. 44, 134 (1984)

[11] R. Widenhorn, M. Fitzgibbons, and E. Bodegom. J. Appl. Phys. 96, 7379 (2004)

[12] Y. F. Chen and S. F. Huang Phys. Rev. B 44, 13775 (1991)

[13] A. Yelon, B. Movaghar and R. S. Crandall Rep. Prog. Phys. 69, 1145 (2006)

[14] A. Yelon, B. Movaghar and H. M. Branz, Phys. Rev. B 46, 12244 (1992)

[15] A. Yelon, Monatsh. Chem. 144, 91 (2013)

[16] J. Olea, D. Pastor, A. del Prado, I. Mártil and G. González-Díaz. J. Phys. D: Appl. Phys. 46, 135108 (2013)

[17] Luque A, Martí A, Antolín E, Tablero C Physica B 382 (2006) 320-327

[18] D. Pastor, J. Olea, A. del Prado, E. García-Hemme, R. García-Hernansanz and G. González-Díaz. Sol. Ener. Mat. And Solar Cells. 104, 159 (2012)

[19] J. Olea, G. González-Díaz, D. Pastor, I. Mártil, A. Martí, E. Antolín, and A. Luque. J. Appl. Phys. 109, 063718 (2011)

[20] J. Olea, D. Pastor, M. Toledano, I. Mártil and G. González-Díaz. Springer Series in Optical Sciences 165, 321 (2012) (A. B. Cristóbal, A. Martí, A. Luque, Editors) Springer, Heidelberg

[21] J. Olea, D. Pastor, A. del Prado, E. García-Hemme, I. Mártil and G. González Díaz. Thin Solid Films 520,6614 (2012)

[22] J. Olea, M. Toledano-Luque, D. Pastor, E. San-Andrés, I. Mártil and G. González-Díaz. J. Appl. Phys. 107,103524 (2010)

[23] J. Mathews, A. J. Akey, D. Recht, G. Malladi, H. Efstathiadis, Michael J. Aziz and Jeffrey M. Warrender. Appl. Phys. Lett. 104, 112102 (2014)

[24] D. Pastor, J. Olea, E. García-Hemme, I. Mártil, G. González Díaz, J. Ibáñez, R. Cuscó and L. Artús, Semicond. Sci. Technol 26, 115003 (2011)

[25] D. Pastor, J. Olea, I. Mártil, G. González-Díaz, A. Muñoz Martín and A. Climent Font. J. Appl. Phys. 112,113514 (2012)

[26] Sharma, S. K.; Sagar, P.; Gupta, Himanshu, Rajendra Kumar and R.M. Mehra Sol. State. Electron. 51, 1124 (2007)

[27] L. Pichon, A. Mercha, J.M. Routoure, R. Carin, O. Bonnaud, T. Mohammed-Brahim. Thin Solid Films 427, 350 (2003)

[28] S. Witanachchi, H. Abou Mourad, and P. Mukherjee J. Appl. Phys. 99, 073710 (2006)

[29] R. Widenhorn, L. Mündermann, A. Rest, and E. Bodegom J. Appl. Phys. 89, 8179 (2001)

[30] Godet C (1994) Philos. Mag. B 70:1003 